\title{
Carbon Paste Electrodes
}

\author{
by \\ Ralph N. Adams \\ Department of Chemistry, University of Kansas, Lawrence, Kansas, USA
}

(Received Nov. 5, 1962)

In the course of examining new electrode systems for anodic oxidations, the carbon paste electrode was developed ${ }^{1)}$. This electrode has proven far more valuable than originally expected and it is the purpose of this paper to critically review its properties, applications, and short-comings. It is fitting that emphasis be placed on applications to organic electrode mechanism studies. One can only hope that future work with this electrode applied to organic electro-oxidations will approach the value of the elegant and classical studies of Professor Tachi and his students in the area of organic reduction processes.

The carbon paste itself consists of a mixture of carbon or graphite mixed with an organic liquid to a typical consistency of "peanut butter". This type of thick paste is tamped into a well-like depression in a Teflon or glass holder and electrical contact is made through the back end of the paste with a platinum or copper wire. A typical form of the electrode has been shown ${ }^{2}$. Other electrode configurations are easily designed but the inverted pool type seems to work very satisfactorily for stationary voltammetry.

The choice of carbon or graphite is dictated primarily by the particle size so that a smooth surface paste results. Graphite GP 38 from National Carbon Company (this is identical with Acheson Grade 38 Graphite which is no longer available) has been found very satisfactory. Typical decolorizing charcoals are, in general, too gritty to give a smooth surface.

The main requirements of the organic liquid are that it not be electroactive or contain electroactive impurities (in the region of potential where the electrode will be used) and that it be highly immiscible with the polarographic solution. To date the most useful liquid is ordinary Nujol mineral oil (used without purification) but carbon tetrachloride, bromoform, bromonaphthalene, etc., have been used with good results. If electrodes are to be stored, the volatility of the pasting liquid is important. The pastes are normally prepared in 10-20 gram batches and stored in sample jars.

All of the liquids mentioned above work very well with aqueous solutions. Unfortunately we have been unable to devise as yet a totally satisfactory carbon paste for use in non-aqueous media such as acetonitrile, N,N-dimethylformamide, etc. The problem would appear simple-find another liquid which is immiscible with these solvent systems. However, pastes prepared from silicones, high molecular weight paraffins, "non-aqueous" stopcock greases, etc., all tend to disintegrate to a degree in the non-aqueous solvents. In view of the normal reproducibility of re-packing a fresh electrode, it is possible to operate in acetonitrile with a new electrode for each run. Some recent pastes made with mixed chloro-fluorohydrocarbons seem to show promise for use in acetonitrile. 
For brevity the various carbon paste electrodes are designated as-carbon electrode$\mathrm{Nu}$ jol paste (CE-NjP), etc, in the following discussions.

\section{Anodic Voltammetry}

The utility of carbon paste electrodes for anodic voltammetry has been firmly established $^{2,3)}$. One of the most valuable features of a CE-NjP is the almost total absence of residual current on the anodic side. Using ordinary voltage scan rates, the residual current line in most backgrounds is almost coincident with zero current and extends, practically flat, out to the onset of the background oxidation. This is delightful contrast to the usual residual current patterns of platinum or gold electrodes. Further, this behavior is largely independent of the previous history of the electrode (except where a previous run has deposited an organic film on the surface). Starting with fresh electrode surfaces, no pretreatment techniques are needed in anodic voltammetry. While it is true that low residual currents can be obtained with wax impregnated carbon rods, the wax impregnation is far more time consuming and involves more experimental "know-how" than the preparation of carbon pastes. Fundamentally, the two types of electrodes behave in a similar manner. The advantage of the carbon paste is the simplicity of preparation plus more consistent low residual current behavior. In addition, the reproducibility of fresh surface areas on a wax impregnated graphite rod requires more attention to detail than do carbon pastes.

For analytical applications it is safe to say that reproducibility of peak currents within $\pm 2-3 \%$ can be obtained routinely after a little experience with preparing smooth paste surfaces. The reproducibility of succesive runs on a given system at a single electrode is usually of the order of a few tenths of a percent. Analytical determinations of not too complex organic mixtures appear feasible but the applications to electrode mechanism studies are of greatest interest.

A study of a very involved and intriguing organic electro-oxidation, that of N,Ndimethylaniline (DMA) in acidic, aqueous buffers, was initiated at platinum electrodes in our laboratory by a student from the Kyoto University polarographic group, T. Mizoguchi $^{4)}$. After the overall process had been established an intensive study was continued at both platinum and carbon paste electrodes ${ }^{5}$. The oxidation pathway of DMA at platinum and carbon paste was found to be identical. Further, the apparent rate constants for DMA and other irreversible organic oxidations like N-methylaniline appear to be quite similar, ${ }^{5,6}$.

The technique of cyclic voltammetry as introduced by Kemula and coworkers ${ }^{7,8)}$ has proven very useful. Details of the application to carbon paste electrodes including controlled potential instrumentation suitable for pen and ink recording have been given. $^{9-11}$ Cyclic voltammetry is probably one of the most useful electroanalytical tools for obtaining an overall, qualitative picture of a complex electrode reaction. It gives a large amount of information with a minimum of time and effort. This is well illustrated by the following example at platinum or carbon paste electrodes.

In $1 N$ sulfuric acid-sodium sulfate buffer, triphenylmethane dyes like crystal violet $(\mathrm{CV})$ and malachite green (MG) oxidize at fairly positive potentials around $+0.8 \mathrm{v} . v s$ SCE. Using a $2 \mathrm{v} . / \mathrm{min}$. triangular wave cyclic sweep, no evidence of any oxidation process other than the primary one at $c a$. $+0.8 \mathrm{v}$. is obtained for CV on the first anodic sweep. On the first return (cathodic) branch, a reduction process appears at $+0.55 \mathrm{v}$. Now, 
on the second anodic and all subsequent sweeps, an almost reversible redox system appears at this lower anodic potential of $+0.55 \mathrm{v}$. From previous experience with the DMA process, this redox couple was suspected of being due to the presence of $\mathrm{N}, \mathrm{N}^{\prime}$ tetramethylbenzidine (TMB). Cyclic polarograms of this system in the same medium show the half-peak potentials of the redox couple formed from CV agree within \pm 2 millivolts with those of the system TMB and its fully oxidized diquinoid TMBOx. Thus CV undergoes a rather remarkable anodic oxidation - the "methane" carbon and one substituted phenyl are lost and two others couple to form the $\mathrm{TMB}^{12)}$. This process had been established for more vigorous chemical oxidations ${ }^{13}$. The electrochemical case can be further substantiated by additional electrochemical and electron spin resonance studies and the details have been examined ${ }^{14}$. While it is true that techniques like reversecurrent chronopotentiometry give the same kind of information, we believe the interpretation of cyclic voltammetry is far less involved. It is certain that cyclic voltammetry led to a much quicker understanding of the triphenylmethane dye oxidation pathway than could have been obtained by most other means.

\section{Cathodic Voltammetry and Anodic Stripping}

The cathodic range of an electrode like the GE-NjP extends to about - 1.4 v. excluding strong acid media. This is, of course, not competitive with mercury, but is a decided advantage over noble metal electrodes. Accordingly, a comprehensive study was made of the potentialities of the $\mathrm{CE}-\mathrm{NjP}$ for reduction processes ${ }^{15}$.

In contrast to the almost non-existant residual current on the anodic side, carbon paste electrodes show a small but non-removable residual current in the cathodic range of potentials. This appears as a small drawn-out wave around -0.4 to $-0.6 \mathrm{v}$. (ca. 1-2 нa for ordinary sized electrodes). This residual current is decreased upon prolonged deaeration or pre-electrolysis but apparently cannot be completely removed. The $\mathrm{pH}$ dependence, decrease upon deaeration, and the reduction potential suggest the residual wave is due to oxygen, dissolved in the paste or strongly sorbed from the polarographic solutions. Other impurities in the paste have not been ruled out of consideration.

Although it cannot be removed completely, this residual current has a very flat, drawn out plateau. It was found that, after usual deaeration procedures, the wave was small and flat enough that reductions could be carried out "on top" of the plateau. The flat portion is simply used as the base line for measuring $i_{p}$. Although low level determinations are precluded, the residual current is of little consequence if one works with $\mathrm{m} M$ concentrations.

Typical of metal ion reductions that can be carried out are Bi(III) in perchlorate and chloride background, Cd(II) in chloride (plots of $i_{p}$ vs $C$ showed satisfactory linearity), $\mathrm{Sn}$ (II) in hydrochloric acid, etc. Somewhat unusual is the fact that Co(II) reduces to the metal in sodium perchlorate and ammonia-ammonium chloride media, but no reduction waves were obtained in hydrochloric or perchloric acids or in $1 M$ potassium chloride. The $\mathrm{Co}(\mathrm{II})$ reduction wave had poor reproducibility. $\mathrm{Fe}(\mathrm{II})$ and $\mathrm{Ni}(\mathrm{II})$ can also be reduced to the metals at the CE- $\mathrm{Nj}$ P.

Following reductions, the diffusion layer was destroyed by moving the electrode and then an anodic stripping polarogram was run to verify metal deposition. It is interesting to note that the $E_{p / 2}$ for $\mathrm{Ni}(\mathrm{II})$ reduction is $-0.97 \mathrm{v}$. vs $\mathrm{SCE}$ whereas the anodic stripping occurs at $c a .+1.0$ v. vs SCE. The observation of such a process illustrates the wide range 
of potentials available with carbon paste electrodes.

As could be anticipated, the $E_{p / 2}$ values for metal ion reductions at the CE-NjP do not agree with those at mercury. While it may have no significance, it appears that those metals which ordinarily form proper amalgams with mercury, show reduction potentials at the CE-NjP more cathodic than at mercury. However, $\mathrm{Co}(\mathrm{II})$ and $\mathrm{Fe}(\mathrm{II})$, which form poor amalgams, if at all, reduce on the $\mathrm{CE}-\mathrm{NjP}$ at more anodic potentials. Further study of these points are required for any definitive statements. Also $E_{p / 2}$ values and peak polarogram shapes at carbon pastes are not as reproducible and well defined as one would prefer. With $\mathrm{Bi}(\mathrm{III})$, for instance, a small "history" effect was noted. If the same electrode is used for several runs, $E_{p / 2}$ shifted slightly for several runs and then stabilized. A more detailed study of these cathodic processes at carbon paste electrodes is required. However, the practical utility of the electrodes for metal ion reductions is evident.

Although the reduction of many organic functional groups is precluded by the limited cathodic range of the $\mathrm{CE}-\mathrm{NjP}$, materials like aliphatic and aromatic nitro compounds are easily handled. Typically, $p$-chloronitrobenzene $\left(E_{p / 2}=-0.42\right.$ v. vs SCE in $\left.1 M \mathrm{HCl}\right)$ and 2-nitro-propane $\left(E_{p / 2}=-1.0 \mathrm{v}\right.$. vs SCE in $\left.1 \mathrm{M} \mathrm{HCl}\right)$ gave irreversible peak polarograms but all indications were that the results would be satisfactory for analytical purposes.

\section{Rotated Disk Electrodes of Carbon Paste}

The rotated disk electrode (RDE) is unique in that the equations for the limiting current at the disk surface can be derived rigorously from hydrodynamic theory and, surprisingly, can be realized experimentally. Carbon paste electrodes function very well when used in a rotated disk configuration.

The usual Teflon plug carbon paste electrode can be converted to a rotated disk by attaching the Teflon holder to a rod which functions as a shaft for the rotator. Ring-disk electrodes are also readily fabricated. The utility of these disk electrodes for analytical voltammetry, diffusion coefficient measurements and electrode mechanism studies has been presented ${ }^{5,16}$.

Most recently carbon paste RDE's were used to determine heterogeneous rate constants of some fairly rapid electron transfer processes. Here the RDE is used to provide a high rate of mass transport so that the charge transfer process becomes current limiting. A system which appears "reversible" at low mass transfer rates shows increasing "irreversibility" as the mass transfer increases and the value of the heterogeneous rate constant, $k_{s}$, can be evaluated. Values of $k_{s}$ for the systems $\mathrm{Fe}(\mathrm{III}) / \mathrm{Fe}(\mathrm{II}), \mathrm{Ce}(\mathrm{IV}) / \mathrm{Ce}(\mathrm{III}), \mathrm{MnO}_{4}^{-}$ $/ \mathrm{Mn}^{++}, \mathrm{Fe}(\mathrm{CN})_{6}{ }^{-3} / \mathrm{Fe}(\mathrm{CN})_{6}^{-4}$ and others were measured and were in essentially excellent agreement with data obtained by other electrochemical techniques ${ }^{17)}$. The simplicity of preparation of carbon paste RDE's seems to offer considerable advantage.

\section{Nature of Charge Transfer at Carbon Paste Electrodes}

The recent study mentioned above examined in detail the measurement of $k_{s}$ values for moderately rapid electrode processes. RDE's of platium and carbon paste were used and it was shown that very reliable data could be obtained with both types of electrode material.

A surprising finding was that the rates at carbon paste were only slightly slower 
than those for the same system at platinum. Table 1 summarizes $k_{z}$ values at the two electrodes and includes some rate constants for organic oxidations evaluated at $E=0$ vs the hydrogen electrode. These latter systems are highly irreversible. Since no formal

Table 1. Comparison of $k_{s}$ Values at Platinum and Carbon Paste Electrodes

\begin{tabular}{|c|c|c|c|c|c|}
\hline System & Background & $\mathrm{Pt}$ & $\begin{array}{c}k_{s}(\mathrm{~cm} / \mathrm{sec}) \\
\mathrm{Ce}-\mathrm{NjP}\end{array}$ & Graphite & $\underset{k_{s}(\mathrm{Pt}) / k_{s}(\mathrm{CE})}{\text { Ratio }}$ \\
\hline $\mathrm{Fe}(\mathrm{III}) / \mathrm{Fe}(\mathrm{II})$ & $1 \mathrm{M} \mathrm{H}_{2} \mathrm{SO}_{4}$ & $4.3 \times 10^{-3}$ & $5.4 \times 10^{-5}$ & \multirow{4}{*}{$6 \times 10^{-3}(b)$} & 80 \\
\hline Ferricy/Ferrocy & $1 M \mathrm{KGl}$ & $9 \times 10^{-2}(a)$ & $7.1 \times 10^{-3}$ & & 13 \\
\hline & $0.5 M \mathrm{~K}_{2} \mathrm{SO}_{4}$ & $1.3 \times 10^{-1}(a)$ & & & 22 \\
\hline $\mathrm{Ce}(\mathrm{IV}) / \mathrm{Ce}(\mathrm{III})$ & $1 M \mathrm{H}_{2} \mathrm{SO}_{4}$ & $\mid \begin{array}{c}3.7 \times 10^{-4} \\
4.5 \times 10^{-4}(c)\end{array}$ & $3.8 \times 10^{-4}$ & & $\begin{array}{c}0.97 \\
(1.18)\end{array}$ \\
\hline DMA & $\begin{array}{l}0.045 M \mathrm{H}_{2} \mathrm{SO}_{4}, \\
0.5 M \mathrm{Na}_{2} \mathrm{SO}_{4}\end{array}$ & $\begin{array}{c}k \text { at } E= \\
6.3 \times 10^{-20}\end{array}$ & $\begin{array}{l}0 v s \mathrm{NHE} \\
2.2 \times 10^{-21}\end{array}$ & & 28 \\
\hline MA & $0.5 \mathrm{MNa}_{2} \mathrm{SO}_{4}$ & $5.0 \times 10^{-18}$ & $2.0 \times 10^{-19}$ & & 25 \\
\hline
\end{tabular}

a) J.E.B. Randles and K.W. Somerton, Trans. Far. Soc., 48, 938 (1952).

b) A. Regner and J. Balej, Coll. Czech. Chem. Comm., 26, 237 (1961), on plain graphite (not wax-impregnated)

c) K. Vetter, Z. physik. Chem., 196, 360 (1950).

potential can be evaluated $k_{s}$ cannot be stated. Nevertheless the mechanism of electrooxidation of $\mathrm{N}, \mathrm{N}$-dimethylaniline (DMA) and $\mathrm{N}$-methylaniline (MA) is similar at platinum and carbon paste, and it is probably safe to make the individual comparison of respective rates. Other unpublished studies show that a variety of organic oxidations proceed with quite similar rates on platinum and carbon paste. For example, the oxidation of hydroquinone, $p$-phenylenediamine, benzidine and substituted benzidines like $\mathrm{N}, \mathrm{N}$-tetramethylbenzidine and $o$-dianisidine all exhibit essentially reversible or quasireversible behavior at both electrodes at relatively high rotation velocities. (These comparisons do not imply that all electrode reactions proceed at comparable rates on both electrodes-the studies to date are limited).

In any event, the difference in rates between the two electrodes is surprisingly small for the system studied. For the $k_{s}$ values which have been measured (Table 1) one can assume for purposes of discussion that $k_{s}$ at carbon paste is about 30-50 times smaller than at platinum. The organic film in the paste ordinarily might be suspected of offering a far greater impedance to charge transfer processes. To examine this point in more detail, the differential capacities of a series of carbon paste electrodes were investigated and correlated with charge transfer rates. The differential capacities were measured in $1 M$ sulfuric acid by the square wave charging curve technique of Hackerman and coworkers. The experimental techniques were identical with those used by these workers on platinum electrodes ${ }^{18}$. As a test system the differential capacity of platinum was checked and the results were in accord with previous data.

Figure 1 is a plot of the differential capacity, $C$, of a variety of carbon paste electrodes $v s$ applied potential. In general these plots are similar to other electrode systems although the small hump at $c a .+0.4 \mathrm{v}$. may be due to a trace of electroactive impurity present in the pastes. Excluding, for the moment, curve 6, the important point is that all pastes 


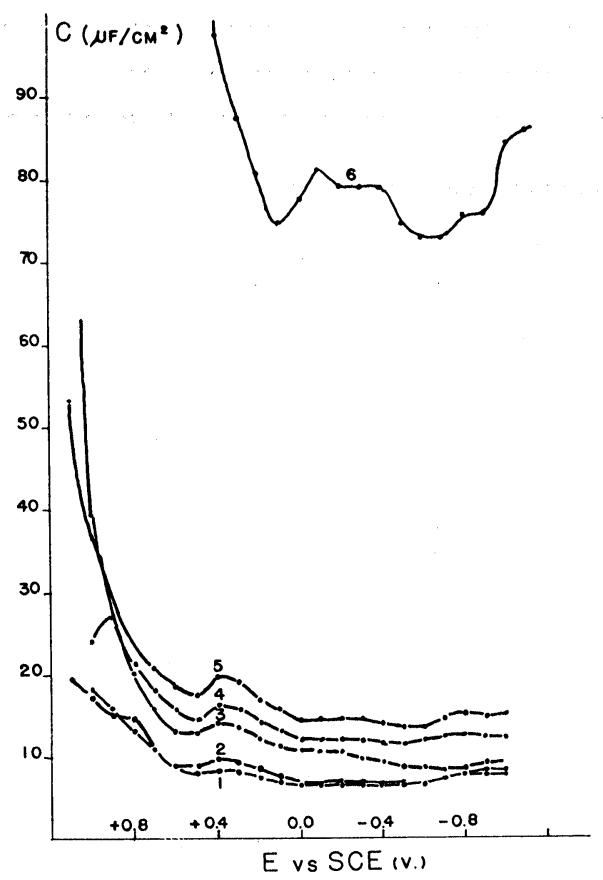

Fig. 1. Differential Capacity vs Applied Potential for Various Carbon Pastes

1. Bromonaphthalene

2. Nujol

3. Bromoform

4. Silicone 1000

5. Silicone 10

6. Silicone 10 in $0.1 \mathrm{M} \mathrm{NaOH}-20 \%$ methanol.

show a capacity quite a bit smaller than platinum. The possibility exists that the organic liquid in a carbon paste electrode really does not cover the carbon particle surface but only wets it in some way which gives cohesive properties but leaves the surface essentially plain carbon. In this case the electrode should have a capacity at least equal to that of platinum on a basis of $C$ per unit area. Obviously $C$ appears to be too small for this type of "uncovered" surface. For instance, $C$ at platinum is of the order of $20-40 \mu \mathrm{F} / \mathrm{cm}^{2}$. It is true that nothing is known of surface roughness factors for carbon paste, but this does not alter the above conclusion. It appears that the carbon particles are indeed covered by the layer of organic liquid.

However since it has been shown that $k_{s}$ values at carbon paste are only slightly different than at platinum, one concludes the bonding forces between carbon and organic liquid are relatively weak.

In Table 2 are listed the values of $C$ for the various electrodes (all measured at 0.0 v. vs SCE in $1 M$ sulfuric acid). In column 2 of this table are shown the $k_{s}$ values for the $\mathrm{Fe}(\mathrm{III}) / \mathrm{Fe}$ (II) system at these

electrodes. These were measured in hydrochloric acid media but it was observed that $C$ was similar in chloride media-certainly the order of $C$ with respect to type of carbon paste remained the same. It is easily seen that as $C$ increases so does $k_{s}$. This parallelism

Table 2. Influence of Paste Composition on $k_{s}{ }^{a}$. Correlation with Differential Capacity

\begin{tabular}{l|l|cc}
\hline \multicolumn{2}{c|}{ Paste Composition $^{b)}$} & \multicolumn{1}{|c|}{$k_{s}(\mathrm{~cm} / \mathrm{sec})$} & $\left.C\left(\mu \mathrm{F} / \mathrm{cm}^{2}\right)^{c}\right)$ \\
\hline Bromonaphthalene & $9.4 \times 10^{-5}$ & 6.55 \\
Nujol & $2.1 \times 10^{-4}$ & 6.74 \\
Bromoform & $3.1 \times 10^{-4}$ & 10.54 & \\
Silicone 1000 & $5.4 \times 10^{-4} \quad k_{s}$ & 11.93 & $C$ \\
Silicone 350 decreasing & $5.8 \times 10^{-4}$ increasing & - & increasing \\
Silicone 50 $\downarrow$ & $6.0 \times 10^{-4} \quad \downarrow$ & - & $\downarrow$ \\
Silicone 20 & $7.3 \times 10^{-4}$ & - & \\
Silicone 10 & $8.2 \times 10^{-4}$ & 14.32 & \\
\hline
\end{tabular}

a) For system $\mathrm{Fe}(\mathrm{III}) / \mathrm{Fe}(\mathrm{II})$ in $0.1 \mathrm{M} \mathrm{HCl}, 3.9 \mathrm{M} \mathrm{NaCl}$ in all cases

b) All pastes consist of $5 \mathrm{gm}$. of Acheson graphite plus $3 \mathrm{ml}$ of indicated organic liquid-except for bromonaphthalene paste where $2 \mathrm{ml}$ of liquid used.

c) Capacity by square wave technique of Hackerman, et al, all measured in $1 \mathrm{M}$ $\mathrm{H}_{2} \mathrm{SO}_{4}$ at 0.0 v. vs $\mathrm{SCE}$. 
might be expected. The larger $C$, the smaller the thickness of the surface film (or the less the coverage) and presumably the smaller the hindrance to the electron transfer reaction. In the case of the silicones 1000 and 10 , silicone 10 with lowest viscosity (i.e., smaller chain length molecules) has the lesser film thickness (or coverage) and hence $C$ is higher. Also, $k_{s}$ is greater. Thus is appears that the variation of $k_{s}$ with $C$ is reasonable.

Evidence that the interaction between carbon and the organic liquid is relatively weak is afforded by curve 6 of Figure 1. Here $C$ was measured for silicone 10 paste in $0.1 M$ sodium hydroxide containing $20 \%$ methanol. The large capacity suggests the silicone film was being dissolved (as can be expected in this medium) and that the electrode surface was more or less "uncovered" carbon with a much higher capacity.

To further check this point the reduction of nitrobenzene in $1 M$ sodium hydroxide was examined. In this reduction at stationary mercury electrode the presence of even small amounts of surface active compounds like camphor cause a marked splitting of the usual 4-electron wave into two l-electron and 3-electron waves ${ }^{8}$. This type of behavior is readily observed with Nujol carbon pastes in alkaline media. However, using the silicone 10 paste in alkaline media, the split character of the nitrobenzene reduction was hardly visible when the polarogram was recorded only a very short time after dipping the electrode in the solution. After 2 minutes immersion the polarogram showed only one wave whose slope and $E_{p / 2}$ agreed quite well with that obtained under similar conditions at a hanging mercury drop electrode (no surface active materials present).

In conclusion it may be said that the limited data available for carbon paste electrodes indicate that the carbon surface is, indeed, covered by a layer of the organic liquid. This layer is only weakly bound to carbon and represents only a relatively small impedance to the charge transfer processes studied so far. Further investigations of a variety of systems are necessary to fully interpret the mechanism of charge transfer at carbon paste electrodes.

\section{Acknowledgements}

The work reported herein is a summary of several years' study by the following coworkers whose contributions are wholeheartedly acknowledged: C. Olson, T. Mizoguchi, H. Y. Lee and D. Walker. The studies of Z. Galus in applying carbon pastes to electrode mechanism studies and the rotating disk were especially significant. The work was supported in part by Research Corporation, the General Research Fund of the University of Kansas, and the Atomic Energy Commission through contract AT(11-1)-686 and this support is gratefully acknowledged.

\section{References}

1) R.N. Adams, Anal. Chem., 30, 1576 (1958).

2) C. Olson and R.N. Adams, Anal. Chim. Acta, 22, 582 (1960).

3) T.R. Mueller, C. Olson, and R.N. Adams, Advances in Polarography, Vol. 1, p. 198. (Proc. 2nd Intn'l. Pol. Cong., Cambridge 1959), Pergamon Press, 1960.

4) T. Mizoguchi and R.N. Adams, J. Am. Chem. Soc., 84, 2058 (1962).

5) Z. Galus and R.N. Adams, ibid., 84, 2061 (1962).

6) Z. Galus and R.N. Adams, submitted for publication, J. Am. Chem. Soc.

7) W. Kemula and Z. Kublik, Nature, 182, 793 (1958).

8) W. Kemula, Advances in Polarography, Vol. 1, p. 105 (Proc. 2nd Intn'l. Pol. Cong., Cambridge 1959), 
Pergamon Press, 1960.

9) G. Olson, H.Y. Lee and R.N. Adams, J. Electroana. Chem., 2, 396 (1961).

10) Z. Galus, H.Y. Lee and R.N. Adams, ibid., 5, 17 (1963).

11) J.R. Alden, J.Q. Chambers, and R.N. Adams, ibid., 5, 152 (1963).

12) Z. Galus and R.N. Adams, J. Am. Chem. Soc., 84, 3207 (1962).

13) V. Hanousek and M. Matrka, Coll. Czech. Chem. Comm., 24, 16 (1959).

14) Z. Galus and R.N. Adams, unpublished data.

15) G. Olson and R.N. Adams, Anal. Chim. Acta, submitted for publication.

16) Z. Galus, G. Olson, H.Y. Lee and R.N. Adams, Anal. Chem., 34, 164 (1962).

17) Z. Galus and R.N. Adams, Abstracts of Papers, 7T, Division of Physical Chemistry, 142nd Meeting, ACS, Atlantic City, N.J., September, 1962.

18) J.J. McMullen and N. Hackerman, J. Electrochem. Soc., 106, 341 (1959). 